\title{
EVALUATION VON LEHRE UND LERNEN
}

\section{REVIEW ARTICLE}

PINTO, Jacyguara Costa ${ }^{1}$

RODRIGUES, Helton Barbosa ${ }^{2}$

SILVA, Vanessa de Souza da ${ }^{3}$

PINTO, Jacyguara Costa. RODRIGUES, Helton Barbosa. SILVA, Vanessa de Souza da. Bewertung von Lehre und Lernen. Revista Científica Multidisciplinar Núcleo do Conhecimento. 04-Jahr, Ed. 11, Band 03, S. 81-90. November 2019. ISSN: 24480959, Zugangslink: https://www.nucleodoconhecimento.com.br/bildungde/lehrbewertung

\section{ZUSAMMENFASSUNG}

Der Artikel stellt Studien über die neuen Herausforderungen für die Bildung vor, und es ist wichtig, über das Bewertungsmodell nachzudenken, das heute von Lehrern verwendet wird. Deshalb haben wir versucht, die Ingelaufungsplanungsprozesse in den Anfangsstufen der Grundschule zu analysieren. Die Ergebnisse wurden durch den Einsatz der bibliographischen Forschungsmethode der beschreibenden Natur und des

\footnotetext{
${ }^{1}$ Doutor em Ciência da Educação, Mestre em Ciência da Educação, Especialista Lato Sensu em Docência de Ensino Superior, Especialista Lato Sensu Em História cultura, Literatura Africana, afro-brasileira, licenciado e Bacharel em História.

${ }^{2}$ Mestre em Ciência da Educação, Licenciatura Plena e Bacharel em Geografia, Especialista Lato Sensu em Gestão Ambiental, professor da Rede pública estadual, atualmente exercendo suas funções de docente na educação básica no Sistema Organizacional ensino Modular do Estado do Amapá.

${ }^{3}$ Mestrando em Ciência da Educação, Especialista em ensino especial, Licenciada plena pedagogia, professora da rede pública Estadual e Municipal no Estado do Amapá.
} 
qualitativen Ansatzes erzielt, die die Analyse von Inhalten von Büchern und wissenschaftlichen Artikeln durchführte, die es ermöglichten, die Studien und empirischen Perspektiven des Forschers miteinander $\mathrm{zu}$ verbinden, und die Konstruktion der Ergebnisse in drei Abschnitten leiteten, die von diesem Punkt an beschrieben wurden. In der Entwicklung des Artikels wurde die Bewertung von Lehre und Lernen analysiert und die Bedeutung der Selbsteinschätzung für den unparteiischen Pädagogen beschrieben; und, kurz gesagt, konnte die Annahme der partizipativen Bewertung durch Ziele in der pädagogischen Realität der Grundschule verstanden werden. Es wurde der Schluss gezogen, dass das Problem des Artikels beantwortet wurde, das allgemeine Ziel erreicht wurde und es wurde die Hypothese bestätigt, dass jedes Instrument, das von Lehrern und der Schule zur Durchführung der Bewertung verwendet wird, eine Möglichkeit darstellt, Lernniveaus zu etablieren, das Beste und das Schlimmste zu erkennen und vor allem den Unterricht an die von der Schule installierten Exzellenzstandards anzupassen. Standardisierte Maßnahmen haben den demokratischen, kritischen, konstruktiven und kreativen Bewertungssinn abgelenkt und den Weg des effektiven Lernens verhindert.

Schlagworte: Bewertung, Kompetenzen, Leistung, Ziele, Schule.

\section{EINFÜHRUNG}

Die Bildung in unserem Land ist recht komplex, die Segregation der Gesellschaft hat Gruppen von Menschen erschüttert, die Opfer von Ungleichheiten sind, die die Widerspiegelung der Inkurien der öffentlichen Macht sind.

Diese Unterschiede spiegeln die Beziehungen wider, die in der Schule bestehen. Den Einzelnen dazu zu bringen, seine Verhältnisse in Frage zu stellen, als ein Wesen, das in der Lage ist, seine Vorstellungen von Mensch, Gesellschaft und Welt zu konstruieren, um zu versuchen, seine Bedürfnisse in verschiedenen Dimensionen zu befriedigen und gleichzeitig seine Identität vor gleichaltrigen Zuteilen zu legitimieren.

Angesichts der neuen Herausforderungen, die sich auf die Bildung stellen, ist es wichtig, über das Bewertungsmodell nachzudenken, das von den Lehrern heute 
verwendet wird, wenn man bedenkt, dass der Akt der Bewertung, unabhängig von dem angenommenen Modell, nicht neutral ist, weil er eine gewisse Art und Weise in seine Ausbuchtung bringt, die Realität, den Einzelnen und die Gesellschaft zu konzipieren, indem er die pädagogische Praxis im Klassenzimmer und in der Schule leitet und konditioniert.

Unabhängig davon, welches Instrument von Lehrern und der Schule für die Evaluierung verwendet wird, konfiguriert es bestimmte, richtet Lernniveaus ein, erkennt die besten und schlechtesten und passt den Unterricht vor allem an die von der Schule installierten Exzellenzstandards an. Standardisierte Maßnahmen haben den demokratischen, kritischen, konstruktiven und kreativen Bewertungssinn abgelenkt und den Weg des effektiven Lernens verhindert. Basierend auf diesen Annahmen beginnt die Untersuchung mit folgendem Problem: Welcher Zusammenhang besteht zwischen den Planungsmaßnahmen der Schulbewertung und der Verbesserung der pädagogischen Leistungsfähigkeit des Schülers?

Daher ist es gerechtfertigt, über die Prozesse nachzudenken, die die Schulbewertungsplanung als Lernmoment beinhalten, ein Mittel, mit dem der Lehrer Wissen vermittelt und nicht ein Ende, da die Bewertung nicht nur auf die Bestimmung der Noten beschränkt ist, sondern den Weg geht, den der Schüler geht, seine Schwierigkeiten entdeckt und die Richtungen ändert. falls erforderlich.

Ziel des Artikels war es daher, die Intestierungsplanungsprozesse in den Anfangsklassen der Grundschule zu analysieren. Insbesondere soll die Bewertung von Lehre und Lernen analysiert werden; beschreiben, wie wichtig die Selbsteinschätzung für den unparteiischen Pädagogen ist; und, um die Annahme der partizipativen Bewertung durch Ziele in der pädagogischen Realität der Grundschule zu verstehen.

Die Ergebnisse wurden durch den Einsatz der bibliographischen Forschungsmethode der beschreibenden Natur und des qualitativen Ansatzes erzielt, die die Analyse von Inhalten von Büchern und wissenschaftlichen Artikeln durchführte, die es ermöglichten, die Studien und empirischen Perspektiven des Forschers miteinander 
zu verbinden, und die Konstruktion der Ergebnisse in drei Abschnitten leiteten, die von diesem Punkt an beschrieben wurden.

\section{2. ÜBER TEACHING UND LEARNING ASSESSMENT}

Nach der Zeit und dem Modell der Gesellschaft hat die Bewertung ihren Verlauf seit den frühesten Zeiten. Einige Autoren wie Soeiro und Aveline (1982) haben als Ursprung der Bewertungsmethoden die Aktivitäten entlarvt, die die ersten menschlichen Konglomerate durch Rituale durchführten, um die jungen Indigenen bereit zu machen, ein indigener Erwachsener zu sein. Für diese Autoren gehen solche Praktiken auf 360.Bc zurück, die als ein System von Kriterien angesehen werden, die von den Chinesen und Griechen verwendet werden, um Einzelpersonen für eine bestimmte Arbeit zu ermöglichen, die es allen Bürgern ermöglichen, Prestige- und Machtpositionen zu erlangen.

Von Anfang an gab es bereits Möglichkeiten zu bewerten, ein Akt, der von den Chinesen und Griechen auf dem Arbeitsmarkt verwendet wurde, geschah dies unbewusst, aufgezwungen durch das Kulturmodell der Gesellschaft, mit dem Ziel, die besten vorzubereiten und auszuwählen, um die Positionen des Prestiges und der Macht zu halten. Dieses Bewertungsmodell wurde auch in China im Jahr 2200 v. Chr. verwendet. (SOEIRO; AVELINE, 1982).

In Griechenland schlug Sokrates eine Selbsteinschätzung vor, "kenne dich selbst", um zur Wahrheit zu gelangen. So ist dieses philosophische Denken zu einer Form der Prüfung geworden, d.h. zur Selbsteinschätzung, zu einer Handlung von extremer Bedeutung, die derzeit für die Wirksamkeit des Lehr-Lern-Prozesses von extremer Bedeutung ist.

Um den Verlauf der Bewertung stärker zu betonen, berichtet Despresbiteris (1999, S. 113), dass in[...] " 2005 BC. Der chinesische Kaiser Shum untersuchte seine Offiziere alle drei Jahre mit dem Ziel, zu fördern oder zu feuern, in erster Linie, um Männer zu sichern, die in der Verteidigung des Staates ausgebildet wurden." Ein weiteres wichtiges Beispiel dafür, wie die Bewertung entwickelt wurde, ist in Marx' Werken, 
$\mathrm{d}[\ldots]$ ass "die Prüfung nichts anderes ist als die bürokratische Taufe des heiligen Wissens" (apud GARCIA, 2001, S. 29).

So wird die Prüfung als Bürokratie betont, d.h. als der schriftliche Teil, in dem nur das berichtet wird, was den Applikatoren angemessen ist, in diesem Fall ist sie auf Dokumentation und nicht auf geistige Entwicklung ausgerichtet, indem das Alltäglichfürzliche für Veränderungen und Transformationen zu heiligem Wissen verwendet wird.

Auch im dreizehnten Jahrhundert entlarvt Kraemer (2005) die Verwendung der Prüfung als eine Form der Bewertung durch die organisierte Disziplin der Prüfungen und das System der Zuweisung von Noten, was zu einer Wissenschaft namens Docimologia. Sie erklärt auch, dass im etymologischen Sinne die Bewertung aus dem Lateinischen $a+$ valere kommt, das als Bedeutung die Zurechnung von Wert und Verdienst zum Studienziel bedeutet. Das in mehreren Ländern bereits erwähnte Konzept zielt darauf ab, Personen auszuwählen, die verschiedene Funktionen auf dem Arbeitsmarkt erfüllen.

Mit den Transformationen aus dem technologischen Zeitalter und der Industrialisierung, ab dem 20. Jahrhundert, litten Forschung und Bewertung unter großen Einflüssen aus den Wissenschaften, zahlreiche Verfahren und Theorien, die heute verwendet werden, wie die Umfrage und ${ }^{[4]}$ andere Instrumente: standardisierte Ziele und schriftliche Tests.

Vianna (2005) weist darauf hin, dass die ersten dreißig Jahre des amerikanischen gesellschaftlichen Lebens im 20. Jahrhundert von drei Elementen beeinflusst wurden, die im Prinzip für das industrielle Management entwickelt wurden: Systematisierung, Standardisierung und Effizienz, die schließlich die gesamte Gesellschaft, auch im Bildungsbereich, betrafen. So gab es eine große Sorge mit dem Arbeitsmarkt, Effizienz war das große Ziel und folglich entwickelt Techniken, um zu identifizieren, wo die Schüler nicht in der Lage waren, das wissen im Klassenzimmer gelehrt zu erfassen. 
In den 1970er Jahren schuf die Bewertung ein positivistisches Paradigma, das mit dem Aufbau neuer Bewertungsformen gebrochen werden sollte, indem die Neuheiten, die auf dem nordamerikanischen Kontinent in Mode waren, angewendet wurden. So wurden Verfahren entwickelt, um die Leistung der Schüler mit Tests und die Zusammensetzung neuer Bildungspläne zu vergleichen, einschließlich der Bewertung als Verfahren zur Messung dieser Leistung in den Disziplinen. Für Prado de Sousa (1998) war es ab den 1980er Jahren, dass die pädagogische Evaluation im schulischen Kontext relevanter wurde. Das war, als die Soziologie begann, relevante Forschung und Studien über das Innenleben der Schule beizusteuern.

Das Konzept des Lernens als Bewertungsobjekt ist eine weitere Säule bei der Verteidigung eines Konzepts der pädagogischen Evaluation. Das menschliche Lernen beschränkt sich nicht auf den Erwerb von Wissen, Fähigkeiten, Verhaltensnormen, "aseptischen" Werten und persönlichem Sinn. Die Verwendung des Konzepts des Lernens, kognitive Prozesse und Akquisitionen zu beschreiben, ohne "affektiv" und "konver" ist inkonsistent, weil sie die Realität verzerrt, die beabsichtigt ist, affektive und evaluierende unbekannte Dimension des Wissens. Jede kognitive Handlung hat Wert, Wissen, Geschicklichkeit, Form des Verhaltens trägt einen persönlichen Sinn, es ist ein Thema, das lernt. Die Evaluierung erfordert, dass das Objektmodell aus einer ganzheitlichen, ganzheitlichen und facettenreichen Perspektive stammt, die die Komplexität und den Reichtum des Lernens hervorhebt. Sie argumentieren, dass die Notwendigkeit einer Bewertung der Lehre oder Ausbildung (GIBBS, 2003) besteht.

\section{DIE BEDEUTUNG DER SELBSTEINSCHÄTZUNG FÜR DEN UNPARTEIISCHEN PÄDAGOGEN}

Die Selbstbewertung wurde als eine sehr effektive Strategie für die Annahme demokratischer Konzepte im Schulmanagementprozess angesehen, die Bürger, Manager, Lehrer und die gesamte Schulgemeinschaft an der Zuweisung von Ressourcen für die Schulbildung beteiligt (CAMILLONI, 1997).

Das Schema der Selbsteinschätzung besteht darin, die internen und externen Prozesse, an denen die Schule teilnimmt, rationell vermessen und die Effizienz der Art 
und Weise demonstrieren zu können, wie die Erofeonik und Didaktik ihre pädagogische und didaktische Planung in den Unterrichtsstrategien im Klassenzimmer analysieren kann, so dass es selbstbewertet wird, wenn man Stärken (Potenzen) und zerbrechlich (Ephemeriden) in den Ergebnissen erkennen kann.

Die Probleme der Bewertungsthemen besteht darin, sich der Debatte darüber zu nähern, wer an der Bewertung teilnehmen soll, kann und kann. Sie bekämpft und verteidigt die Notwendigkeit und das Recht der Studierenden, an ihrer Bewertung teilzunehmen, den Bildungs- und Ausbildungswert, der sich selbst trägt, und die anderen Themen des Lehr-Lern-Prozesses. Das Selbst und Hetero sind notwendige Facetten der Einzel- und Gruppenarbeit im Hochschulbereich, die die Beziehung zwischen der Arbeit der Gruppe und der individuellen Verantwortung jeder besonderen Würdigung der Gruppe verstärken und zum Ausdruck bringen; um zur Entwicklung der postgradualen sozialen Verantwortung als Voraussetzung für die Hochschulbildung beizutragen. Es wird als legitimer Zweck festgelegt, der den bewerteten Bewerter umkehrt.

Die Selbstbewertung erfolgt durch Prozesse, bei denen, wenn der Lehrer sie durchführt und annimmt, keine Kenntnisse über die Informationen und Kriterien haben, die bewertet werden müssen, weil sie von denjenigen durchgeführt wurden, die sie bewerten, ihre eigenen Ergebnisse messen und prüfen, ob solche eigenen Ergebnisse für den Erfolg des Lernprozesses des Unterrichts zufriedenstellend waren. In diesem Fall wenden Lehrer Selbstbewertungstests an, wenn Kurse, Vorlesungen, Schulungen und Beratung im Klassenzimmer und in anderen Schulräumen unterrichtet werden.

Die pädagogische Bewertung, seit ihrer Entstehung, bringt bereits in ihrer Ausbuchtung eine Komplexität, aufgrund der zahlreichen Faktoren, die bei der Bewertung von jemandem oder etwas berücksichtigt werden sollten, und es ist wichtig zu betonen, dass es heutzutage ein Bewusstsein der brasilianischen Gesellschaft über die Relevanz des bildungspolitischen Bewertungsprozesses gibt.

Daher ist eine Evaluierung notwendig, und zwar kontinuierlich, umso mehr, wenn die Evaluierung als formelle Verpflichtung und informelle Praxis angesehen wird. Da die 
Bewertung derzeit als eine unverzichtbare Handlung im Leben des Menschen verstanden wird, die sich natürlich im Dienste der Bildung stellt, sich den Lernerfahrungen, der menschlichen Entwicklung, der Verbesserung der Lebensqualität, dem Wohlwollen, der Erhöhung des Selbstwertgefühls und der Aufwertung von Initiativen unter den Menschen stellt.

Die Gewährleistung der Bildung für alle hängt mit dem Wandel von einem Curriculum fernab der Probleme der Welt und den Interessen der Schüler selbst zu einem Curriculum zusammen, das in der Lage ist, das Interesse sowohl der Schüler als auch der Lehrer zu erfassen und das Verständnis natürlicher, sozialer und kultureller Phänomene zu fördern, als ein Instrument, das dazu dient, die Einstellung, der Unsicherheit der Komplexität zu trotzen, in Frage zu stellen, zu lernen, in Aktion zu lernen. , unter Berücksichtigung der neuen Kenntnisse, Fähigkeiten und Werte.

Der Pädagoge muss seine Praxis und Didaktik neu bewerten, um einen bedeutenderen Inhalt und eine partizipativere Methodik zu entwickeln, so dass die Notwendigkeit, die Note als Instrument der Zwangzulage zu verwenden, reduziert wird. Diese Aufgabe ist im aktuellen Kontext nicht einfach, diese Leistung des Pädagogen hat mit der Bewältigung der Entfremdung zu tun: Es ist ein Kampf der Perspektiven, der Bedeutungen für Wissen und für das Leben.

In diesem Zusammenhang zielt die LDB als rechtlicher Leitfaden für bildungspolitische Maßnahmen darauf ab, durch die öffentliche Schule inre Rolle bei der Bildung der Bürgerschaft wiederherzustellen, ohne Diskriminierung allen Schülern offen zu stehen, soziokulturelle Vielfalt und individuelle Unterschiede zu integrieren und so zu einer integrativen Sozialisierung beizutragen. Die Erziehung der Staatsbürgerschaft weist auf einen Weg hin, neue Probleme zu bewältigen, während gleichzeitig die Integration von Werten und Querschnittsthemen ermöglicht wird, anstatt sie als spezifische oder isolierte Aktionen darzustellen, ohne sich auf das Erlernen bestimmter Werte, Verhaltensweisen oder Einstellungen zu beschränken, da der Bürger das gesamte Wissen und die Fähigkeiten benötigt, die es inm ermöglichen, aktiv am öffentlichen Leben teilzunehmen. , ohne die Sie ausgeschlossen oder die Staatsbürgerschaft verweigert werden können. 
Es gibt jedoch noch viele Herausforderungen, denen sich die öffentliche Bildung stellen muss, um eine Voraussetzung für den sozialen Zusammenhalt und die soziale Integration zu sein: mehr Effizienz im Management und mehr Inhaltlichkeit; Dialog zwischen wissenschaftlichen Erkenntnissen, den Interpretationssystemen der Welt und der uralten Weisheit der lateinamerikanischen Völker; offene Schulen für die Gesellschaft und ihre Bedürfnisse; Definition der grundlegenden und grundlegenden Fähigkeiten für die Ausbildung der Studierenden mit der Schaffung von Informationsund Bewertungssystemen; größere soziale Anerkennung für das Lehramt, würdeende und professionalisierende Pädagogen.

\section{VERWENDUNG DER PARTIZIPATIVEN BEWERTUNG NACH ZIELEN IN DER BRASILIANISCHEN GRUNDBILDUNG}

Im aktuellen Kontext wird die Bewertung, die wir haben, immer noch manchmal mit Konzepten und Noten verwechselt und durchgeführt, um die Schüler in genehmigt und abgelehnt zu klassifizieren. Es gibt Minderheiten, die sich die Bewertung vorstellen und durchführen können, um zur Qualifizierung des Bildungsprozesses beizutragen. Die meisten ziehen es vor, durch die Noten zu sortieren, weil sie denken, es ist der einfachste Weg, dass so werden sie weniger Arbeit haben.

Viele Schulen bewerten ihre Schüler anhand von Prüfungen, Praktiken, die positivistischer und technischer Natur sind und das Lernen nicht als Ganzes betrachten, indem sie klassifikativ handeln und ausschließen, denn obwohl Noten/Konzepte aufgrund des aktuellen Bildungssystems eine notwendige Aufzeichnung sind, können sie die Bewertung selbst nicht darstellen und den LehrLern-Prozess verzerren. Bei dieser Art der Bewertung, die klassifiziert, ist das am meisten gesunde das Produkt. Reflektierend, eine Ausbildung, die auf dem Auswendiglernen von Inhalten basiert.

Im Gegensatz zu dieser Art der Bewertung, die die Zuordnung von Noten und Klassifikationen betont, kann die qualitative Bewertung nachgewiesen werden, die auf einem kritischen Paradigma basiert und darauf abzielt, die Qualität des Bildungssystems zu verbessern. In der Erwägung, dass der Schwerpunkt auf dem 
Prozess der Überwindung der Schwierigkeiten der Studenten liegt. Und das ist die Einschätzung, die derzeit angesichts so vieler Herausforderungen, die die Schule täglich durchmacht, gewünscht wird.

Derzeit herrscht Einigkeit über die neuen Bewertungsmethoden, die im Lehrprozess der Grundbildung umgesetzt werden. Diese Neuheit im Bereich der Pädagogik hat die Teilnahme der Schüler hervorgebracht und mit Informationen und Meinungen beigetragen, die die Transformation von Wissen und Lehre fördern können, und gipfelt in der Verbesserung der Qualität des Unterrichts, indem beispielsweise die partizipative Bewertung durch Ziele, Bewertungsstrategien, die Meinungen und persönliche Visionen - kritisch oder überwindend - der Strategien, die Lehrer im Klassenzimmer angenommen haben, übereinstimmen.

Diese Technik wurde verwendet, um greifbare Ergebnisse der von den Lehrern in Betracht gezogenen Ziele und pädagogischen Planungen zu identifizieren, auch Punkte im Zusammenhang mit den Leistungsbildern (Verbesserung oder Verschlechterung) der Schüler zu identifizieren und ihre Meinungen zu identifizieren, wenn Änderungen in den derzeitigen pädagogischen Praktiken angenommen werden. Laut Kraemer (2005) hat diese Praxis zwei wichtige Funktionen, mit ihren kurzen Beschreibungen in Diagramm 1.

Tabelle 1. Klassifizierung der Bewertungsfunktionen nach Zielen.

\begin{tabular}{|l|l|}
\hline Klassifizierung & Funktionen und Eigenschaften \\
\hline Diagnosefunktion & $\begin{array}{l}\text { Unter seinen Aufgaben heben wir die Meinungen } \\
\text { derjenigen hervor, die beispielsweise in Bezug auf die } \\
\text { Verwaltung von Lehrerlehrstrategien bewertet werden; } \\
\text { oder auch die Perspektiven der Schüler auf die Ursachen } \\
\text { der schlechten Leistung im Klassenzimmer in einer } \\
\text { bestimmten Disziplin. Diese Art der Datenerhebung } \\
\text { schlägt den Einsatz innovativer, gewagter und kreativer } \\
\text { Vortests, Umfragen und Strategien (Hirnsturm) vor, die } \\
\text { darauf abzielen, bei der Diagnose des Unterrichts und der }\end{array}$ \\
\hline
\end{tabular}




\begin{tabular}{|l|l|}
\hline & $\begin{array}{l}\text { Übernahme der Kultur der Veränderungen in den } \\
\text { vorhandenen pädagogischen Strategien behilflich zu sein. }\end{array}$ \\
\hline $\begin{array}{l}\text { Summationsfunktion } \\
\text { oder Klassifikation }\end{array}$ & $\begin{array}{l}\text { Zu seinen Hauptmerkmalen gehört die Annahme von } \\
\text { ausgearbeiteten Tests oder die Einstufung des Schülers } \\
\text { am Ende des Schuljahres (genehmigt oder abgelehnt) } \\
\text { klassifizieren und charakterisieren können. Es war die am } \\
\text { meisten akzeptiert in den Schulen für die synthetische } \\
\text { DieErstellung der Wahrnehmungen über die Schulrealität } \\
\text { im Klassenzimmer und am Ende der Bimaster und } \\
\text { Semester. }\end{array}$
\end{tabular}

Fonte: Kraemer (2005).

Mit diesem Ansatz lässt sich daraus schließen, dass die Bewertung der Schulleistung als Zuschreibung einer Qualität zu den Lernergebnissen der Schüler auf der Grundlage ihrer wesentlichen Aspekte praktiziert werden muss und dass als Endziel eine Entscheidungsfindung bevorzugt wird, die das Lernen und damit die Entwicklung des Schülers leitet.

So ist die diagnostische Auswertung als ein Moment des Verständnisses der Phase konfiguriert, in der der Schüler von der Distanz ist, in der er auf die artikulierten Ziele verweist, die vor uns erreicht werden müssen. Die diagnostische Bewertung begünstigt auch die gesellschaftliche Teilhabe aller Schüler als Bürger, da sie nicht mehr den konservativen und undemokratischen Charakter der Klassifizierungsbewertung hat, die viele Menschen von der Schule ausschloss.

Unter dieser Prämisse zielt die diagnostische Bewertung darauf ab, die Schwierigkeiten und Ziele zu identifizieren, zu überprüfen, zu untersuchen, die erreicht wurden, um die Bildungsmaßnahme zu ändern, und es ist ein Verfahrensakt, der den Pädagogen dazu bringt, über die Ergebnisse seiner Forschung nachzudenken, neue Vorschläge, Methoden zu entwickeln, um Qualität und Engagement zu lehren, und die Notwendigkeit, den Schüler kontinuierlich zu bewerten. , um die verschiedenen 
Momente seiner Entwicklung zu erkennen, nicht auf das Urteil über den Erfolg oder Misserfolg der Schüler beschränkt, es als eine Reihe von Leistungen zu verstehen, die die Funktion der Führung pädagogischer Intervention hat, geschieht dies kontinuierlich und systematisch durch die qualitative Interpretation von Wissen, das vom Studenten konstruiert wurde.

Der Lehrer wird die Bewertung als Instrument nutzen, das es inm ermöglicht, seine Vorschläge im Lehr-Lernprozess ständig in Frage zu stellen, so dass der Pädagoge seine Praxis umleiten kann.

\section{FAZIT}

Es wird angenommen, dass das Problem des Artikels beantwortet wurde, das allgemeine Ziel erreicht wurde und es wurde die Hypothese bestätigt, dass jedes Instrument, das von Lehrern und der Schule verwendet wird, um die Bewertung durchzuführen, bestimmte konfiguriert, Lernniveaus festlegt, das Beste und das Schlimmste erkennt und vor allem den Unterricht an die von der Schule installierten Exzellenzstandards anpasst. Standardisierte Maßnahmen haben den demokratischen, kritischen, konstruktiven und kreativen Bewertungssinn abgelenkt und den Weg des effektiven Lernens verhindert.

Auch die Ziele wurden erreicht. In Bezug auf das Ziel der Analyse der Bewertung von Lehre und Lernen, die Autoren analysiert enmittiert die Herkunft der Bewertungsmethoden und ihre Verwendung durch die Chinesen und Griechen auf dem Arbeitsmarkt. Das Zeichen des dreizehnten Jahrhunderts war die Organisation von Evaluierungsprüfungen mit der Zuteilung von Noten, was zu einer Wissenschaft namens Docimologia führte. Heute wurden Verfahren entwickelt, um die Leistung der Schüler mit Tests und die Zusammensetzung neuer Bildungspläne zu vergleichen, einschließlich der Bewertung als Prozess zur Messung dieser Leistung in den Disziplinen.

In Bezug auf das zweite spezifische Ziel, das vorgeschlagen wurde, die Bedeutung der Selbsteinschätzung für den unparteiischen Pädagogen zu analysieren, wurde in 
den ausgewählten Studien festgestellt, dass das Selbstbewertungsschema darin besteht, dass die internen und externen Prozesse, an denen die Schule beteiligt ist, ihre Ergebnisse rational messen lassen und die Effizienz für die Art und Weise demonstrieren, in der ihre pädagogische und didaktische Planung zufriedenstellende Ergebnisse in den Unterrichtsstrategien im Unterricht erzielt hat. So gibt es eine Selbsteinschätzung, wenn man Stärken (Potenzen) und zerbrechlich (Ephemeriden) in den Ergebnissen identifizieren kann.

Schließlich wurde das dritte spezifische Ziel erreicht, das zeigt, dass die Verwendung der partizipativen Bewertung nach Zielen in der brasilianischen Grundbildung zu einer Art und Weise führt, die dem der Bewertung zuwiderläuft, die Noten und Klassen gibt. Der Schwerpunkt liegt auf dem Prozess der Überwindung der Schwierigkeiten der Studenten. Und das ist die Einschätzung, die derzeit angesichts so vieler Herausforderungen, die die Schule täglich durchmacht, gewünscht wird. Diese Technik wurde verwendet, um greifbare Ergebnisse der von den Lehrern in Betracht gezogenen Ziele und pädagogischen Planungen zu identifizieren und auch Punkte im Zusammenhang mit den Leistungsbildern (Verbesserung oder Verschlechterung) der Schüler zu identifizieren.

\section{REFERENZEN}

CAMILLONI, A. R. W. Los obstáculos epistemológicos em la enseñanza. Barcelona: Gedisa, 1997.

DEPRESBITERIS, Léa. O desafio da avaliação da aprendizagem: dos fundamentos a uma proposta inovadora. $1^{\text {a }}$ ed. São Paulo: Editora Pedagógica e Universitária, 1999.

GARCIA, Ronaldo Coutinho. Subsídios para organizar avaliações da ação governamental. Planejamento e Políticas Públicas, Brasília, n. 23, p. 7-70, jan. /jun. 2001. 
GIBBS, G. Uso estratégico de la evaluación en el aprendizaje. In: Brown, S.; Glasner, A. Evaluar em la Universidad: problemas y nuevos enfoques. Madrid: Narcea, 2003, pp. 61-70.

KRAEMER, Maria Elisabeth Pereira. A avaliação da aprendizagem como processo construtivo de um novo fazer. Avaliação: Revista da Avaliação da Educação Superior, v. 10, n. 2, 137-147, 2005.

SOEIRO, L \& AVELINE, S. Avaliação Educacional. Porto Alegre: Editora. Sulina, 1982.

VIANNA, Heraldo Marelim. Fundamentos de um Programa de Avaliação Educacional. Brasília: Liber Livro Editora, 2005.

4. Testes normativos usados para avaliar a eficiência da Instituição escolar.

Veröffentlicht: September, 2019.

Genehmigt: November 2019. 